\title{
Semidiscrete quantum droplets and vortices
}

\author{
Xiliang Zhang ${ }^{1, a}$, Xiaoxi $\mathrm{Xu}^{1, a}$, Yiyin Zheng ${ }^{1}$, Zhaopin $\mathrm{Chen}^{2}$, Bin \\ $\mathrm{Liu}^{1}$, Chunqing Huang ${ }^{1}$, Boris A. Malomed ${ }^{2,1}$, and Yongyao Li ${ }^{1,2}$ * \\ ${ }^{1}$ School of Physics and Optoelectronic Engineering, Foshan University, Foshan 528000, China \\ 2 Department of Physical Electronics, School of Electrical Engineering, Faculty of Engineering, \\ and the Center for Light-Matter Interaction, Tel Aviv University, Tel Aviv 69978, Israel. \\ ${ }^{a}$ These two authors contributed equally to this paper.
}

\begin{abstract}
We consider a binary bosonic condensate with weak mean-field (MF) residual repulsion, loaded in an array of nearly one-dimensional traps coupled by transverse hopping. With the MF force balanced by the effectively one-dimensional attraction, induced in each trap by the Lee-Hung-Yang correction (produced by quantum fluctuations around the MF state), stable onsite-centered and intersite-centered semidiscrete quantum droplets (QDs) emerge in the array, as fundamental ones and self-trapped vortices, with winding numbers, at least, up to 5, in both tightly-bound and quasi-continuum forms. The application of a relatively strong trapping potential leads to squeezing transitions, which increase the number of sites in fundamental QDs, and eventually replace vortex modes by fundamental or dipole ones. The results provide the first realization of stable semidiscrete vortex QDs, including ones with multiple vorticity.
\end{abstract}

Introduction and the model. Recent works with binary Bose-Einstein condensates (BECs) have led to a breakthrough in studies of quantum matter, predicting and experimentally realizing ultradilute superfluids which form quantum droplets (QDs). They were predicted in the three-dimensional (3D) setting [1], as well as in its 2D and $1 \mathrm{D}$ reductions [2], on the basis of mean-field (MF) Gross-Pitaevskii equations (GPEs) with the Lee-HungYang (LHY) corrections, that account for quantum fluctuations around MF states [3]. In 3D and 2D geometries, the LHY terms are repulsive, helping to stabilize a binary condensate against the collapse driven by the cross-attraction between its components, which slightly exceeds self-repulsion in each one, the residual attraction being balanced by the LHY terms. As a result, stable multidimensional soliton-like states may be created in the form of QDs, which is a problem of great interest [4], a challenging issue being stability of $2 \mathrm{D}$ and $3 \mathrm{D}$ solitons against the collapse. The prediction was followed by the creation of quasi-2D [5, 6] and isotropic 3D [7, 8] QDs in a binary condensate of two different states of ${ }^{39} \mathrm{~K}$ atoms. The competition of long-range attractive interactions and LHY repulsion has also made it possible to create stable QDs in single-component condensates of dipolar atoms 9]-13]. In addition to their significance to fundamental studies, QDs offer potential applications, such as the design of matter-wave interferometers [14]. An essential extension is the recent prediction of stable 3D [15] and 2D [16] two-component QDs with embedded vorticity and robust necklace-shaped clusters [17] (vortex QDs in dipolar condensates were found to be unstable [18]).

The reduction of the MF system with the LHY corrections to the $1 \mathrm{D}$ configuration (the condensate loaded in a cigar-shaped trap subject to strong transverse confinement [19 22]) changes the setting, making the LHY

*Electronic address: yongyaoli@gmail.com term attractive, on the contrary to its repulsive sign in higher dimensions [2]. Accordingly, the most relevant case is one with the residual cubic MF repulsion (the inter-component attraction being slightly weaker than the repulsion in each component) competing with a quadratic term representing the LHY-induced attraction. Self-trapped states in this model demonstrate Gaussianlike and flat-top shapes in the case of relatively small or large numbers of atoms, respectively [23]. The next natural step is the consideration of a tunnel-coupled pair of $1 \mathrm{D}$ waveguides, in which spontaneous symmetry breaking of QDs was predicted 24] (similar systems, combining the LHY term and linear mixing between two components, were introduced too [25, 26]).

The availability of optical lattices (OLs) for BEC experiments [27, 28] suggests to consider a setting in the form of an array of $1 \mathrm{D}$ traps, coupled by hopping to adjacent ones. Similar configurations were broadly considered in optics, in the form of parallel-coupled arrays of fibers and stacks of planar waveguides, in temporal- and spatial-domain forms, respectively [29]-[40]. In the combination with intra-core nonlinearity, they give rise to $2 \mathrm{D}$ semidiscrete solitons, which are continuous objects along the guiding cores and discrete in the transverse direction [30, 31, 37, 40, 41].

In this work, we aim to introduce semidiscrete QDs in the system of transversely coupled 1D traps, filled by the binary condensate which features the combination of the weak MF repulsion and LHY-induced attraction in each trap. Subjects of special interest are semidiscrete solitary vortices, which were not considered previously. We produce stable solutions for both fundamental (zerovorticity) and vortical semidiscrete QDs, with the winding number up to $S=5$. In the $2 \mathrm{D}$ continuum form, bright vortex solitons were produced in various models [42]-[57], [15, 16], the main issue being their stability [? ]. While the stabilization of vortices was theoretically elaborated in diverse forms, it was demonstrated experimentally only in nonlocal media [60]. In settings with 
local nonlinearity, self-trapped vortices were experimentally observed in transient forms [58, 59]. On the other hand, vortex solitons were predicted in 2D [61, 62] and 3D [63] fully discrete media, including stable 2D modes with $S \geq 2$ [64]. Such 2D discrete states were created in photorefractive lattices [65, 66]. The robustness of semidiscrete QDs presented below, and available techniques for the work with QDs [5]-[8] suggest that the creation of the semidiscrete states is a relevant objective for experiments.

The setting is realized in the form of the system of linearly-coupled GPEs for the semidiscrete wave function, $\psi_{j}(z, t)$ (in the basic form, the same for both components of the binary condensate [1, 2]), with longitudinal coordinate $z$ and the transverse discrete one, $j$. The GPE system includes the cubic self-repulsion competing with the LHY-induced quadratic self-attraction. In a scaled form [2, 23], it is

$$
\begin{aligned}
i \partial_{t} \psi_{j}= & -(1 / 2) \partial_{z z} \psi_{j}-(C / 2)\left(\psi_{j+1}-2 \psi_{j}+\psi_{j-1}\right) \\
& +g\left|\psi_{j}\right|^{2} \psi_{j}-\left|\psi_{j}\right| \psi_{j}+\left(\omega^{2} / 2\right) z^{2} \psi_{j},
\end{aligned}
$$

where $C>0$ is the coupling between adjacent cores, the strength of the quadratic attraction is normalized to be 1 , and $g>0$ is the strength of the cubic self-repulsion. A realistic model should include a trapping potential with strength $\omega^{2}$ (its action in the discrete direction is negligible, as the trapping effect of the OL potential, which makes the setting semidiscrete, is much stronger). Estimates for physical parameters of the system and predicted semidiscrete modes are given below.

It is relevant to mention studies of fully discrete $1 \mathrm{D}$ and 2D solitons [67, 68], which are supported by the competition of cubic-quintic onsite nonlinearities. Similarly, we find many branches of zero-vorticity states, of onsitecentered (OC) and intersite-centered (IC) types, which are chiefly stable, but tend to disappear with the increase of $C$, as the medium is approaching a quasi-continuum (QC) regime. However, in the present work we address semidiscrete modes, rather than fully discrete ones, and we address semidiscrete vortices, with winding numbers $1 \leq S \leq 5$, that were not considered previously.

The total norm, $N=\sum_{j} \int_{-\infty}^{+\infty}\left|\psi_{j}(z)\right|^{2} d z$, is fixed by choosing its particular value. First, for states with vorticities $S=0$ and 1, the fixed value is $N_{S=0,1}=100$, which is appropriate for plotting the results [as shown below, typical values of the actual (unscaled) number of atoms are $\sim 10^{4}$. For $S \geq 2$, it is convenient to fix larger values of $N$. Two remaining control parameters are $C$ and $g$, that will be varied in the range of $0 \leq C, g \leq 1$, which is sufficient for identifying all species of self-trapped states and making conclusions about their stability. Along with the norm, the system conserves the energy,

$$
\begin{aligned}
& E=\sum_{j} \int_{-\infty}^{+\infty}\left\{C\left[\left|\psi_{j}\right|^{2}-\frac{1}{2} \psi_{j}^{*}\left(\psi_{j+1}+\psi_{j-1}\right)\right]\right. \\
& \left.+\frac{1}{2}\left|\left(\psi_{j}\right)_{z}\right|^{2}+\frac{g}{2}\left|\psi_{j}\right|^{4}-\frac{2}{3}\left|\psi_{j}\right|^{3}+\frac{\omega^{2}}{2}\left|\psi_{j}\right|^{2}\right\} d z
\end{aligned}
$$

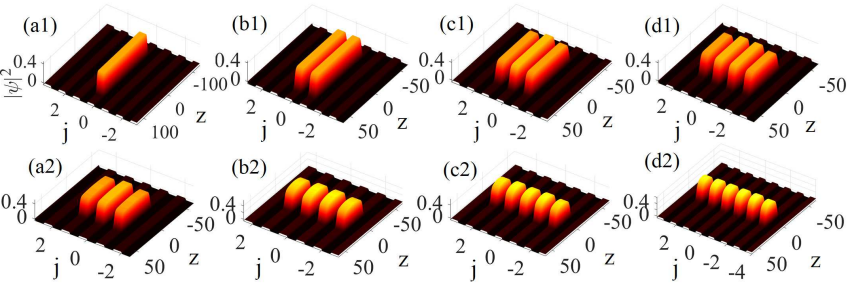

FIG. 1: Density profiles of coexisting stable OC and IC semidiscrete QDs with zero vorticity and the number of sites from 1 to 4 in panels (a1-d1), respectively. Parameters are $\omega=0$ and $(g, C, N)=(1,0.01,100)$. (a2-d2) QDs produced from those displayed in (a1-d1) by the squeezing transition imposed by the trap with $\omega=0.004,0.011,0.015$ and 0.021 in Eq. (1), respectively. The corresponding transition points are $\omega_{c}=0.003,0.010,0.014$, and 0.020 , and the ratio of the QD's longitudinal size to the trapping length, $L_{\omega}=\omega_{c}^{-1 / 2}$, is $L_{z} / L_{\omega}=10.20,8.11,6.74$, and 5.93.

Stationary states with chemical potential $\mu$ are looked for as $\psi_{j}(z, t)=\phi_{j}(z) e^{-i \mu t}$, where $\phi_{j}(z)$ is a localized wave function. In the limit of $C=0$, the uncoupled GPE (11) gives rise to 1D QDs. Particularly, they assume the flat-top shape with a nearly constant density, $|\phi|^{2}=4 /(9 g)$, at $\mu$ close to $\mu_{0}=4 / 9-2 /(3 \sqrt{g})$, at which the QD's width diverges [2, 23]. On the other hand, in the limit of $g \rightarrow 0$ the QD takes a well-localized shape, $\phi_{g=0}(z)=(3 / 2)\left|\mu_{g=0}\right| \operatorname{sech}^{2}\left(\sqrt{\left|\mu_{g=0}\right| / 2} z\right)$, with $\mu_{g=0}=-(1 / 3)\left(2 N^{2} / 3\right)^{1 / 3}$.

The anisotropy of $2 \mathrm{D}$ QDs in the $(z, j)$ plane is defined as the ratio of its widths in the $z$ and $j$ directions, $\varepsilon=\sqrt{C} L_{z} / L_{j}$, with $L_{z} \equiv$ $\left[\int_{-\infty}^{+\infty}\left|\phi_{j=0}(z)\right|^{4} d z\right]^{-1}\left(\int_{-\infty}^{+\infty}\left|\phi_{j=0}(z)\right|^{2} d z\right)^{2}$ and $L_{j} \equiv$ $\left[\sum_{j}\left|\phi_{j}(z=0)\right|^{4}\right]^{-1}\left[\sum_{j}\left|\phi_{j}(z=0)\right|^{2}\right]^{2}$. Indeed, in the continuum limit $(C \rightarrow \infty), \varepsilon=1$ implies that the $2 \mathrm{D}$ mode is axially symmetric in the plane of coordinates $(z, j / \sqrt{C})$. It is shown below that $\varepsilon$ determines a boundary between tightly-bound (TB) and QC semidiscrete states.

To generate stationary modes, Eq. (11) was solved numerically, using the imaginary-time and squaredoperator [70] methods for finding QDs with $S=0$ and $S \geq 1$, respectively. Stability of the stationary states was then identified through computation of eigenvalues for small perturbations, and by dint of simulations of Eq. (11) in real time, both approaches producing almost identical results. The use of the GPE with the LHY term is relevant for exploring stability of states supported by quantum fluctuations, as implied by the derivation of the model [1, 2], and confirmed by direct comparison of the predictions with experimentally observed dynamics [5-7].

Zero-vorticity QDs. Two kinds of zero-vorticity modes, $v i z$., OC and IC ones, which occupy, respectively, $2 J-1$ and $2 J$ sites, are produced by the numerical solution. 

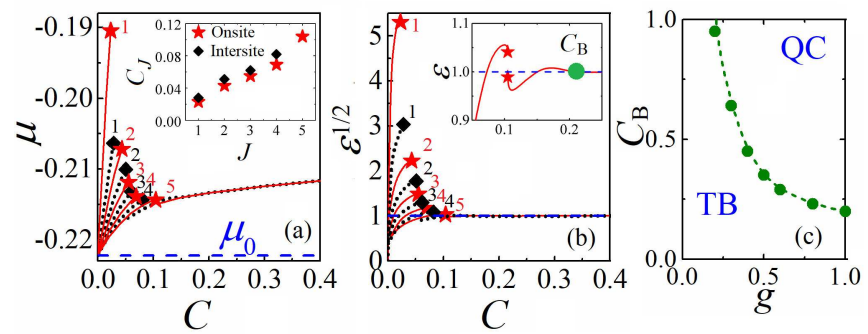

FIG. 2: (a,b) Dependences $\mu(C)$ and $\sqrt{\varepsilon(C)}$ for the OC and IC (red solid and black dotted lines, respectively) QDs with zero vorticity, built of $2 J-1$ or $2 J$ sites, versus $J$, at $g=1$ and $\omega=0$. The dashed line in (a) is $\mu_{0}(g=1) \approx-0.222$. These branches exist up to terminal values of $J$, viz., $J_{\mathrm{T}}^{(\text {on,in })}=5$ and 4 , respectively. The subplot in (a) shows critical values, $C_{J}$, at which the respective branches terminate, vs. $J$. The subplot in (b) displays the switch of the single eventually surviving onsite-centered mode from $J=5$ to 6 with the subsequent increase of $C$. The green point, $C_{\mathrm{B}}$, at which $\varepsilon=1$, is the boundary between the QDs with the TB and QC structure, for $g=1$. (c) The TB-QC boundary, $C_{\mathrm{B}}(g)$.

Starting from the anti-continuum limit $(C \rightarrow 0)$ 71], many coexisting solutions are found, corresponding to $1 \leq J \leq 5$ and $1 \leq J \leq 4$ for the OC and IC configurations, respectively, i.e., with the number of sites from 1 to 9 , see examples of stable semidiscrete modes with $J=1,2$ in Fig. 11. The coexisting solution branches are represented by the respective dependences $\mu(C)$ and $\varepsilon(C)$, for the above-mentioned fixed norm, $N_{S=0,1}=100$, and fixed $g$, in Fig. 2(a,b). The comparison of energy (2) for different modes demonstrates that the ground state (energy minimum) always corresponds to the largest number of sites. For these states, the energy of the intersite coupling is $\sim 2 \%-5 \%$ of the total energy, and $6 \%-8 \%$ for the vortex states considered below.

The branches originate from $\mu=\mu_{0}$ and $\varepsilon=0$ at $C=0$, and terminate at critical values $C=C_{J}$ [69], which are denoted in Figs. 2(a) and (b) by red stars are black rhombuses for the OC and IC states, respectively. The subplot in Fig. 22(a) shows $C_{J}$ is a function of $J$, demonstrating that the multiplicity of coexisting branches reduces, step by step, with the increase of $C$. The single branch survives at $C \geq 0.10$, carrying over into the single fundamental mode with $\varepsilon=1$ in the $2 \mathrm{D}$ continuum, as seen in Fig. 2(b). With the increase of $C$, the evolution of the single surviving state proceeds through the increase of the number of sites in this state. An example is the transition from 9 to 11 sites, with an incremental increase of $C$, as shown in the inset to Fig. 2(b).

Semidiscrete QDs can be categorized as TB or QC ones, if their shapes feature strong or weak discreteness, respectively, the boundary between them being determined by proximity of $\varepsilon$ to 1 . The transition from $\varepsilon \neq 1$ to $\varepsilon=1$ is illustrated by the inset to Fig. 2(b), where $C_{\mathrm{B}}$ is identified as the transition point. A boundary between
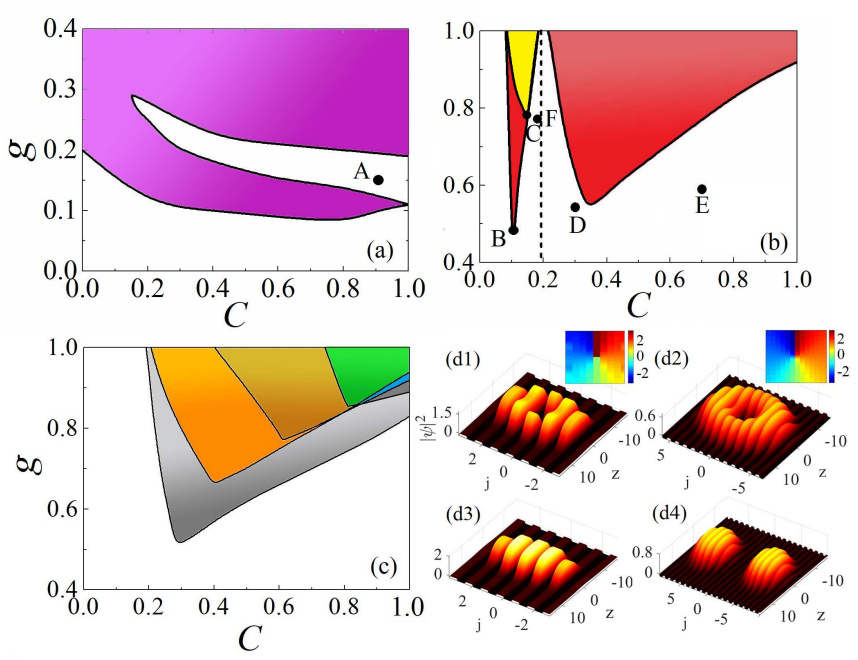

FIG. 3: (a) The stability area (purple) for IC semidiscrete QDs with zero vorticity, in the $(C, g)$ parameter plane. Unstable modes, such as the one corresponding to point A, with $(g, C)=(0.9,0.15)$, transform into robust breathers, as shown in Fig. 4(a). Panel (b) displays stability areas for OC and IC vortex QDs with $S=1$ (yellow and red regions, and the yellow-only one, respectively). In panels (a) and (b), the fixed normalization is $N=100$. (c) Stability areas for OC vortices with $S=2$ (all colored regions), 3 (orange + brown + green $), 4$ (brown + green + blue $)$, and 5 (green + blue + dark gray). For the convenience of plotting, the normalization for $S=2$ through 5 is fixed as $N=400,900,2500$ and 4500 , respectively. (d1,d2) Examples of stable onsiteand intersite-centered vortices, for $(g, C)=(0.48,0.1)$ and $(0.77,0.15)$, which correspond, respectively, to points $\mathrm{B}$ and $\mathrm{C}$ in panel (c). The insets display the respective phase profiles. (d3,d4) Fundamental and dipole-mode QDs, into which the squeezing transforms the onsite- and intersite-centered vortices at $\omega=0.024$ and 0.006 . The corresponding critical values are $\omega_{c}^{\prime}=0.023$ and 0.005 , respectively.

the TB and QC regimes in the $(g, C)$ plane is displayed in Fig. 2(c). The decrease of the boundary value, $C_{\mathrm{B}}$, with the increase of $g$ is a natural trend, as stronger selfrepulsion makes the droplet broader in the discrete direction, which is the same effect as produced by stronger coupling.

Analysis of the stability of the semidiscrete QDs with $S=0$ demonstrates that the OC modes are stable in the entire $(g, C)$ plane, while their IC counterparts are stable only in a part of the plane [Fig. 3(a)]. The instability of the latter states at small $g$, and their stabilization at larger $g$, is similar to findings for $1 \mathrm{D}$ discrete solitons in the model with the cubic-quintic nonlinearity [67]. However, a new feature is an inner lacuna in the stability area. In direct simulations, unstable IC QDs transform into robust breathers, which perform shuttle oscillations [Fig. 4(a)].

With the increase of $\omega$ (the trap's strength), QDs found at $\omega=0$ undergo a squeezing transition at critical values $\omega_{c}$, which transforms them into stable QDs with two 


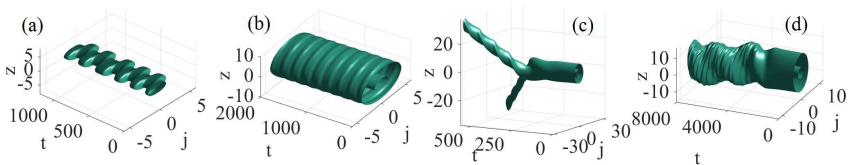

FIG. 4: Examples of the evolution of unstable semidiscrete QDs. (a) The OC QD with $S=0$, denoted by point A in Fig. 3 (a), transforms into a breather which performs shuttle motion. (b,c,d) Vortex QDs with $(g, C)=(0.55,0.3),(0.65,0.6)$ and $(0.75,0.18)$, which are denoted by points D-F in Fig. 3(d).

sites added at their edges, as shown in Fig. 1(a2-d2). For QDs with larger numbers of sites, viz., 5, 6, 7, 8, the respective critical values are $\omega_{c}=0.025,0.038,0.032,0.045$ and $L_{z} / L_{\omega}=5.33,4.94,4.62,4.37$. The large values of the length ratio imply robustness of the QDs against the squeezing.

Vortex modes. Semidiscrete states with vorticity $S$ represent a novel species of self-trapped modes, their stability being a central issue, as suggested by studies of vortex solitons in continuous models [? ]. The present system gives rise to such states at $g \geq g_{\min } \approx 0.4$. They seem as quasi-isotropic modes, with $\varepsilon$ close to 1 . The systematic numerical analysis identifies stability areas for OC vortex QDs with $S=1$ and $2 \leq S \leq 5$, which are displayed in Figs. 3(b) and (c), respectively. For $S=1$, the stability area is split in two parts at $C \approx 0.19$ [by the dashed line in Fig. 31)(b)], approximately equal to value $C_{\mathrm{B}}$ separating the $\mathrm{TB}$ and $\mathrm{QC}$ regions at $g=1$ in Fig. 2(d). Vortices with $S \geq 2$ were found only at $C>0.19$.

There are two different kinds of stable vortices with $S=1$, of the OC and IC types, with pivots located, respectively at a lattice site or between two sites. Stable IC vortices are found only in a small yellow parameter region in Fig. 3(b) at $C<0.19$, where the semidiscrete states feature a TB structure, and they do not exist with $S \geq 2$ (in fully discrete 2 D lattices it is also difficult to find stable IC vortex solitons [68]). Stable OC and IC vortex QDs [points B and C in Fig. 3(a)] are displayed in Fig. $3(\mathrm{~d} 1, \mathrm{~d} 2)$. In the QC region $(C>0.19)$, stability areas for OC vortices with $2 \leq S \leq 5$ are displayed in Fig. 3(c), being similar to their counterpart with $S=1$ in Fig. 3(b). The vortex QDs are stable at values of $g$ exceeding a certain threshold, which gradually increases with $S$.

With the increase of the trap's strength, $\omega$, the squeezing transition leads to destabilization of OC and IC vortices from Figs. 3(d1,d2) at $\omega_{c}=0.005$ and 0.001 , respectively, the corresponding size ratios being $L_{z} / L_{\omega}=0.94$ and 0.60 , i.e., the vortices are more fragile states than the fundamental states. With the further increase of $\omega$, the unstable vortices are replaced by stable fundamental and dipole-mode QDs at $\omega_{c}^{\prime}=0.023$ and 0.005 [Fig. 3 $3(\mathrm{~d} 3, \mathrm{~d} 4)]$, the corresponding length ratios being $L_{z} / L_{\omega} \simeq 1.83$ and 1.42 .

Unstable vortex QDs display different evolution scenarios. Close to the stability boundary, they form robust breathers which keep initial $S$ [Fig. 廿(b)]. Far from the stability boundary, an unstable vortex QD splits in two fragments [Fig. 4(c)]. Near the TB-QC boundary, unstable vortices undergo conspicuous deformation, but do not split, keeping $S$ and featuring chaotic evolution in Fig. 4(d). Its chaotic character is confirmed, following a known criterion [72], by computation of the power spectrum of oscillations of the peak density, in which $28 \%$ of the total power belongs to a continuous component.

Undoing the rescaling used in the derivation of Eq. (1) [1, 2], we conclude that a longitudinal size of the states is expected to be $L_{z} \sim 10 \mu \mathrm{m}$ with $\sim 10^{4}-10^{5}$ atoms of ${ }^{39} \mathrm{~K}[5]$ - [8], transverse trap $\omega_{\mathrm{tr}} \sim 2 \pi \times 200 \mathrm{~Hz}$, and the OL potential with wavelength $\sim 4 \mu \mathrm{m}$ and respective recoil energy, $2 m_{\text {atom }}^{-1}(\pi \hbar / \lambda)^{2}$. The coupling constant, $C$, may be adjusted by variation of the OL depth 73$]$. The critical strength of the longitudinal trap, which initiates the squeezing transition, is $\sim \omega_{\text {tr }}$ for the robust $S=0$ states, while for more fragile vortices it is $\sim 2 \pi \times 20 \mathrm{~Hz}$. Vorticity may be imparted to the condensate by a helical optical beam, transversely focused on spotsize $\sim L_{z}$ 74]. The experimental realization is definitely possible at temperatures $\lesssim 2.5 \mu \mathrm{K}[\underline{6}]$. Due to three-body losses, the modes will start to decay at $t \gtrsim 50 \mathrm{~ms}$, which allows one to observe them by means of available techniques $\left[\begin{array}{ll}5 & 7\end{array}\right]$.

Conclusion. We have introduced a setting for the study of semidiscrete QDs in the form of the array of $1 \mathrm{D}$ guides coupled by hopping of atoms. Each guide is filled by a binary condensate, which gives rise to a semidiscrete system, in the form of the GPE including the repulsive cubic and attractive quadratic (LHY) terms, with the longitudinal continuous and transverse discrete coordinates. The systematic analysis reveals many families of stable 2D semidiscrete QDs, of the onsite- and intersitecentered (OC and IC) types, which terminate one by one with the increase of the coupling coefficient. The system's parameter space splits into tight-binding (TB) and quasi-continuum (QC) parts, with a single stable family surviving in the latter one. Previously unexplored self-trapped modes are semidiscrete vortices. In the TB region, vortex $\mathrm{QDs}$, of both $\mathrm{OC}$ and IC types, are stable with winding number $S=1$, while in the QC region OC vortices remain stable up to $S=5$. The application of the longitudinal trap leads to squeezing transitions of $S=0$ states, and, eventually, to transformation of vortices into fundamental or dipole modes.

A similar setting may be implemented for spatial optical solitons in stacks of planar waveguides with cubicquintic nonlinearity [75]. A challenging extension is to consider a $3 \mathrm{D}$ setting with two discrete coordinates.

\section{Acknowledgments}

This work was supported by NNSFC (China) through Grant No. 11874112, No. 11575063 and No. 11905032, Guangdong Provincial Department of Education Project 
through Grant No. 2018KQNCX279, the Israel Science Foundation through Grant No. 1286/17, and by the special Funds for the Cultivation of Guangdong Col- lege students Scientific and Technological innovation No. pdjh2019b0514
[1] D. S. Petrov, Quantum Mechanical Stabilization of a Collapsing Bose-Bose Mixture, Phys. Rev. Lett. 115, 155302 (2015).

[2] D. S. Petrov and G. E. Astrakharchik, Ultradilute Low-Dimensional Liquids, Phys. Rev. Lett 117, 100401 (2016).

[3] T. D. Lee, K. Huang, and C. N. Yang, Eigenvalues and Eigenfunctions of a Bose System of Hard Spheres and Its Low-Temperature Properties, Phys. Rev. 106, 1135 (1957).

[4] Y. Kartashov, G. Astrakharchik, B. Malomed, and L. Torner, Frontiers in multidimensional self-trapping of nonlinear fields and matter, Nature Reviews Physics 1, 185-197 (2019).

[5] C. R. Cabrera, L. Tanzi, J. Sanz, B. Naylor, P. Thomas, P. Cheiney, L. Tarruell, Quantum liquid droplets in a mixture of Bose-Einstein condensates, Science 359, 301 (2018).

[6] P. Cheiney, C. R. Cabrera, J. Sanz, B. Naylor, L. Tanzi, and L. Tarruell, Bright Soliton to Quantum Droplet Transition in a Mixture of Bose-Einstein Condensates, Phys. Rev. Lett. 120, 135301 (2018).

[7] G. Semeghini, G. Ferioli, L. Masi, C. Mazzinghi, L. Wolswijk, F. Minardi, M. Modugno, G. Modugno, M. Inguscio, and M. Fattori, Self-bound quantum droplets in atomic mixtures, Phys. Rev. Lett. 120, 235301 (2018).

[8] G. Ferioli, G. Semeghini, L. Masi, G. Giusti, G. Modugno, M. Inguscio, A. Gallemí, A. Recati, and M. Fattori, Collisions of self-bound quantum droplets, Phys. Rev. Lett. 122, 090401 (2019).

[9] H. Kadau, M. Schmitt, M. Wentzel, C. Wink, T. Maier, I. Ferrier-Barbut, T. Pfau, Observing the Rosenzweig instability of a quantum ferrofluid, Nature 530, 194-197 (2016).

[10] I. Ferrier-Barbut, H. Kadau, M. Schmitt, M. Wenzel, T. Pfau, Observation of quantum droplets in a strongly dipolar Bose gas, Phys. Rev. Lett. 116, 215301 (2016).

[11] M. Schmitt, M. Wenzel, F. Böttcher, I. Ferrier-Barbut, and T. Pfau, Self-bound droplets of a dilute magnetic quantum liquid, Nature, 539, 259 (2016).

[12] L. Chomaz, S. Baier, D. Petter, M. J. Mark, F. Wächtler, L. Santos, and F. Ferlaino, Quantum-Fluctuation-Driven Crossover from a Dilute Bose-Einstein Condensate to a Macrodroplet in a Dipolar Quantum Fluid, Phys. Rev. X 6, 041039 (2016).

[13] H. Saito, Path-integral Monte-Carlo study on a droplet of a dipolar Bose-Einstein condensate stabilized by quantum fluctuation, J. Phys. Soc. Jpn. 85, 053001 (2016).

[14] B. Laburthe-Tolra, A strange kind of liquid, Nature (London) 539, 176 (2016).

[15] Y. V. Kartashov, B. A. Malomed, L. Tarruell, and L. Torner, Three-dimensional droplets of swirling superfluids, Phys. Rev. A 98, 013612 (2018).

[16] Y. Li, Z. Chen, Z. Luo, C. Huang, H. Tan, W. Pang, and B. A. Malomed, Two-dimensional vortex quantum droplets, Phys. Rev. A 98, 063602 (2018).
[17] Y. V. Kartashov, B. A. Malomed, and L. Torner, Metastability of quantum droplet clusters, Phys. Rev. Lett. 122, 193902 (2019).

[18] A. Cidrim, F. E. A. dos Santos, E. A. L. Henn, and T. Macrí, Vortices in self-bound dipolar droplets, Phys. Rev. A 98, 023618 92018).

[19] M. Olshanii, V. Dunjko, and V. Lorent, Bosons in cigarshaped traps: Thomas-Fermi, Tonks-Girardeau regime, and in between, Phys. Rev. Lett. 86, 5413-5416 (2001).

[20] L. Salasnich, A. Parola, and L. Reatto, Effective wave equations for the dynamics of cigar-shaped and diskshaped Bose condensates, Phys. Rev. A 65, 043614 (2002).

[21] K. E. Strecker, G. B. Partridge, A. G. Truscott, and R. G. Hulet, Bright matter wave solitons in Bose-Einstein condensates, New J. Phys. 5, 73 (2003).

[22] Z. Zhou, X. Yu, Y. Zou, H. Zhong, Dynamics of quantum droplets in a one-dimensional optical lattice, Commun. Nonlinear Sci. Numer. Simulat. 78104881 (2019).

[23] G. E. Astrakharchik, and B. A. Malomed, Dynamics of one-dimensional quantum droplets, Phys. Rev. A 98, 013631 (2018).

[24] B. Liu, H. Zhang, R. Zhong, X. Zhang, X. Qin, C. Huang, Y. Li, B. A. Malomed, Symmetry breaking of quantum droplets in a dual-core trap, Phys. Rev. A. 99, 053602 (2019).

[25] A. Cappellaro, T. Macrí, G. F. Bertacco, and L. Salasnich, Equation of state and self-bound droplet in Rabicoupled Bose mixtures. Sci. Rep. 7, 13358 (2017).

[26] A. Tononi, Y. Wang, and L. Salasnich, Quantum solitons in spin-orbit-coupled Bose-Bose mixtures, Phys. Rev. A 99, 063618 (2019).

[27] M. Lewenstein, A. Sanpera, V. Ahufinger, B. Damski, A. Sen (De), and U. Sen, Ultracold atomic gases in optical lattices: Mimicking condensed matter physics and beyond, Adv. Phys. 56, 243-379 (2007).

[28] I. Bloch, J. Dalibard, and W. Zwerger, Many-body physics with ultracold gases, Rev. Mod. Phys. 80, 885964 (2008).

[29] D. N. Christodoulides and R. I. Joseph, Discrete selffocusing in nonlinear arrays of coupled waveguides, Opt. Lett. 13, 794-796 (1988).

[30] A. B. Aceves, C. De Angelis, A. M. Rubenchik, and S. K. Turitsyn, Multidimensional solitons in fiber arrays, Opt. Lett. 19, 329-331 (1994).

[31] A. B. Aceves, G. G. Luther, C. De Angelis, A. M. Rubenchik, and S. K. Turitsyn, Energy localization in nonlinear fiber arrays: Collapse-effect compressor, Phys. Rev. Lett. 75, 73-76 (1995).

[32] Y. Liu, G. Bartal, D. A. Genov, and X. Zhang, Subwavelength discrete solitons in nonlinear metamaterials, Phys. Rev. Lett. 99, 153901 (2007).

[33] Y. V. Kartashov, V. A. Vysloukh, and L. Torner, Soliton shape and mobility control in optical lattices, Prog. Opt. 52, 63-148 (2009).

[34] F. Ye, D. Mihalache, B. Hu, and N. C. Panoiu, Sub- 
wavelength plasmonic lattice solitons in arrays of metallic nanowires, Phys. Rev. Lett. 104, 106802 (2010).

[35] A. Marini, A. V. Gorbach, and D. V. Skryabin, Coupledmode approach to surface plasmon polaritons in nonlinear periodic structures, Opt. Lett. 35, 3532-3534 (2010).

[36] M. Conforti, C. De Angelis, and T. R. Akylas, Energy localization and transport in binary waveguide arrays, Phys. Rev. A 83, 043822 (2011).

[37] F. Eilenberger, S. Minardi, A. Szameit, U. Röpke, J. Kobelke, K. Schuster, H. Bartelt, S. Nolte, A. Tünnermann, and T. Pertsch, Light bullets in waveguide arrays: spacetime-coupling, spectral symmetry breaking and superluminal decay [Invited], Opt. Exp. 19, 23711-23187 (2011).

[38] T. Utikal, M. Hentschel, and H. Giessen, Nonlinear photonics with metallic nanostructures on top of dielectrics and waveguides, Appl. Phys. B 105, 51-65 (2011).

[39] Y. Kou, F. Ye, and X. Chen, Multipole plasmonic lattice solitons, Phys. Rev. A 84, 033855 (2011).

[40] R. Blit and B. A. Malomed, Propagation and collisions of semidiscrete solitons in arrayed and stacked waveguides, Phys. Rev. A 86, 043841 (2012).

[41] N. C. Panoiu, R. M. Osgood, and B. A. Malomed, Semidiscrete composite solitons in arrays of quadratically nonlinear waveguides, Opt. Lett. 31, 1097-1099 (2006).

[42] M. Quiroga-Teixeiro, H. Michinel, Stable azimuthal stationary state in quintic nonlinear optical media, J. Opt. Soc. Am. B 14, 2004-2009 (1997).

[43] I. Towers, A. V. Buryak, R. A. Sammut, and B. A. Malomed, Stable localized vortex solitons, Phys. Rev. E 63, 055601(R) (2001).

[44] R. L. Pego and H. A. Warchall, Spectrally stable encapsulated vortices for nonlinear Schrödinger equations, J. Nonlinear Sci. 12, 347-394 (2002).

[45] D. Mihalache, D. Mazilu, I. Towers, B. A. Malomed, and F. Lederer, Stable two-dimensional spinning solitons in a bimodal cubic-quintic model with four-wave mixing, J. Optics A 4, 615-623 (2002).

[46] H. Saito and M. Ueda, Split instability of a vortex in an attractive Bose-Einstein condensate, Phys. Rev. Lett. 89, 190402 (2002).

[47] B. B. Baizakov, B. A. Malomed, and M. Salerno, Multidimensional solitons in periodic potentials, Europhys. Lett. 63, 642-648 (2003).

[48] J. Yang and Z. H. Musslimani, Fundamental and vortex solitons in a two-dimensional optical lattice, Opt. Lett. 28, 2094-2096 (2003).

[49] S. K. Adhikari, Mean-field model of interaction between bright vortex solitons in Bose-Einstein condensates, New J. Phys. 5, 137 (2003).

[50] D. Mihalache, D. Mazilu, F. Lederer, Y. V. Kartashov, L.-C. Crasovan, and L. Torner, Stable three-dimensional spatiotemporal solitons in a two-dimensional photonic lattice, Phys. Rev. E 70, 055603(R) (2004).

[51] Y. V. Kartashov, V. A. Vysloukh, L. Torner, Stable ringprofile vortex solitons in Bessel optical lattices, Phys. Rev. Lett. 94, 043902 (2005).

[52] D. Briedis, D. E. Petersen, D. Edmundson, W. Królikowski, and O. Bang, Ring vortex solitons in nonlocal nonlinear media, Opt. Express 13, 435-443 (2005).

[53] A. I. Yakimenko, Y. A. Zaliznyak, and Y. Kivshar, Stable vortex solitons in nonlocal self-focusing nonlinear media, Phys. Rev. E 71 065603(R) (2005).

[54] Y. V. Kartashov, B. A. Malomed, Y. Shnir, and L.
Torner, Twisted toroidal vortex solitons in inhomogeneous media with repulsive nonlinearity, Phys. Rev. Lett. 113, 264101 (2014).

[55] H. Sakaguchi, E. Ya. Sherman, and B. A. Malomed, Vortex solitons in two-dimensional spin-orbit coupled BoseEinstein condensates: Effects of the Rashba-Dresselhaus coupling and Zeeman splitting, Phys. Rev. E 94, 032202 (2016).

[56] J. Qin, G. Dong, and B. A. Malomed, Stable giant vortex annuli in microwave-coupled atomic condensates, Phys. Rev. A 94, 053611 (2016).

[57] S. Gautam and S. K. Adhikari, Vortex-bright solitons in a spin-orbit coupled spin-1 condensate, Phys. Rev. A 95, 013608 (2017).

[58] F. Eilenberger, K. Prater, S. Minardi, R. Geiss, U. Röpke, J. Kobelke, K. Schuster, H. Bartelt, S. Nolte, A. Tünnermann, and T. Pertsch, Observation of discrete, vortex light bullets, Phys. Rev. X 3, 041031 (2013).

[59] A. S. Reyna, G. Boudebs, B. A. Malomed, C. B. de Araújo, Robust self-trapping of vortex beams in a saturable optical medium, Phys. Rev. A 93, 013840 (2016).

[60] Y. Izdebskaya, G. Assanto, and W. Królikowski, Observation of stable-vector vortex solitons, Opt. Lett. 40, 41824185 (2015).

[61] B. A. Malomed and P. G. Kevrekidis, Discrete vortex solitons, Phys. Rev. E 64, 026601 (2001).

[62] D. E. Pelinovsky, P. G. Kevrekidis, and D. J. Frantzeskakis, Persistence and stability of discrete vortices in nonlinear Schrödinger lattices, Physica D 212, 20-53 (2005).

[63] P. G. Kevrekidis, B. A. Malomed, D. J. Frantzeskakis and R. Carretero-González, Three-dimensional solitary waves and vortices in a discrete nonlinear Schrödinger lattice, Phys. Rev. Lett. 93, 080403 (2004).

[64] P. G. Kevrekidis, B. A. Malomed, Z. Chen, and D. J. Frantzeskakis. Stable higher-order vortices and quasivortices in the discrete nonlinear Schrödinger equation. Phys. Rev. E 70, 056612 (2004).

[65] D. N. Neshev, T. J. Alexander, E. A. Ostrovskaya, Y. S. Kivshar, H. Martin, I. Makasyuk, and Z. G. Chen, Observation of discrete vortex solitons in optically induced photonic lattices, Phys. Rev. Lett. 92, 123903 (2004).

[66] J. W. Fleischer, G. Bartal, O. Cohen, O. Manela, M. Segev, J. Hudock, and D. N. Christodoulides, Observation of vortex-ring "discrete" solitons in 2D photonic lattices, Phys. Rev. Lett. 92, 123904 (2004).

[67] R. Carretero-Gonzalez, J. D. Talley, C. Chong, and B. A. Malomed, Multistable solitons in the cubic-quintic discrete nonlinear Schrödinger equation, Physica D 216, 77-89 (2006).

[68] C. Chong, R. Carretero-González, B. A. Malomed, P. G. Kevrekidis, Multistable solitons in higher-dimensional cubic-quintic nonlinear Schrödinger lattices, Physica D 238, 126-136 (2009).

[69] In fact, the branches terminate at the critical points through saddle-note bifurcations generated by collision with additional branches of unstable solutions, which are not shown in the figure.

[70] J. Yang and T. I. Lakoba, Universally-convergent squared-operator iteration methods for solitary waves in general nonlinear wave equations, Stud. Appl. Math 118, 153-197 (2007).

[71] J. L. Marin and S. Aubry, Breathers in nonlinear lattices: Numerical calculation from the anticontinuous 
limit, Nonlinearity 9, 1501-1528 (1996).

[72] M. Lakshmanan and S. Rajaseckar, Nonlinear Dynamics: Integrability, Chaos and Patterns (Springer: Berlin, 2003),

[73] A. Trombettoni and A. Smerzi, Discrete solitons and breathers with dilute Bose-Einstein condensates, Phys. Rev. Lett. 86, 2353-2356 (2001).

[74] R. Pugatch, M. Shuker, O. Firstenberg, A. Ron, and N.
Davidson, Topological stability of stored optical vortices, Phys. Rev. Lett. 98, 203601 (2007).

[75] E. L. Falcão-Filho, C. B. de Araújo, G. Boudebs, H. Leblond, and V. Skarka, Robust two-dimensional spatial solitons in liquid carbon disulfide, Phys. Rev. Lett. 110, 013901 (2013). 